\title{
The arrow of time, complexity and the scale free analysis
}

\author{
Dhurjati Prasad Datta*and Santanu Raut \\ Department of Mathematics, University of North Bengal \\ P.O. Siliguri, West Bengal, Pin: 734430, India
}

\begin{abstract}
The origin of complex structures, randomness, and irreversibility are analyzed in the scale free $\operatorname{SL}(2, R)$ analysis, which is an extension of the ordinary analysis based on the recently uncovered scale free $C^{2^{n}-1}$ solutions to linear ordinary differential equations. The role of an intelligent decision making is discussed. We offer an explanation of the recently observed universal renormalization group dynamics at the edge of chaos in logistic maps. The present formalism is also applied to give a first principle explanation of $1 / f$ noise in electrical circuits and solid state devices. Its relevance to heavy tailed (hyperbolic) distributions is pointed out.
\end{abstract}

Chaos, Solitons and Fractals 28, 581-589, (2006) 


\section{Introduction}

The origin of the arrow of time is still considered to be a puzzling problem in theoretical physics [1]. Another difficult problem is the $1 / f$ noise [2], a footprint of complexity. The ubiquity of $1 / f$-like noise in diverse natural and biological processes seems, in particular, to signal to certain key, but still not clearly understood, dynamical principles that might be at work universally at the heart of any given dynamical process. Recently, there seems to have been an emerging urge in literature [3] for a new principle for understanding complex, intrinsically irreversible, processes in nature.

In this paper, we argue that the recently discovered finitely differentiable scale free solutions to the simplest scale free ordinary differential equation (ODE) [4]-[7]

$$
t \frac{\mathrm{d} \tau}{\mathrm{d} t}=\tau
$$

should be able to offer an ideal framework, analogous to one advocated in Ref.[3] , for complex phenomena. In particular, we reemphasize how both the above problems might acquire a natural explanation in the class of such solutions. Both the problems were originally discussed in [4, 5, 6]. The present discussion based on the new exact class of time asymmetric $C^{2^{n}-1}$ solutions to eq(11) [7] is, however, expected to put the previous analyses in a stronger foundation. The simplest ode (1) will also be shown to enjoy a host of new dynamical features shedding new lights in the definitions of living and nonliving systems. The relevance of the present formalism to complex systems has already been pointed out in literature [8].

To summarize briefly the previous results, we present, in Ref[4], a novel dynamical treatment of linear ODEs when the time (i.e. the independent real) variable $t$ is assumed to have a random element. We show how a judicious use of the golden mean partition of unity, $\nu^{2}+\nu=1, \nu=$ $(\sqrt{5}-1) / 2$, not only allows time to undergo random changes (flips) by inversions, $t_{-} \rightarrow t_{-}^{-1}=$ $t_{+}, t_{ \pm}=1 \pm \eta$, in the vicinity of an instant $t=1$ (say), but also unveils, for the first time, the possible existence of a class of random, second derivative discontinuous, scale free solutions to eq(11). One of the major aims of [4] is to explore the especially unique role of the golden mean number in this extended framework of calculus accommodating inversions as a valid mode of changes (increments) besides ordinary translations. The freedom of random inversions provides a dynamic, evolutionary character to the second derivative discontinuous solutions, with a privileged sense of time's arrow, represented graphically by the slow, progressive unfolding of the approximants of the golden mean continued fraction. We also give a derivation of a universal probability distribution for these intrinsic random fluctuations. These are further clarified in [5] where we treated, in a sense, the 'inverse problem'. We show that the existence of a nontrivial solution of the form $\tau(t)=t(1+\phi(t)), \phi(t)=t_{1} \tau\left(t_{1}^{-1}\right) \neq$ an ordinary constant, $t_{1}=\epsilon t, \epsilon$ being 
a scaling parameter, to eq(11) could only be interpreted in an extended framework of calculus accommodating random inversions as a valid mode of changes for a genuine set of infinitesimals [9]. We also point out how this extended dynamical calculus provides a natural framework for the explanation of the origin of the generic $1 / f$ spectrum. These ideas become more precise and concrete in [6] where we present an explicit solution of the form $\tau\left(t_{-}\right)=1 / t_{+}$to eq(1). This solution, though approximate $\left(\sim \mathrm{O}\left(\eta^{2}\right)=0\right)$, in the ordinary real number system $R$, is, however, exact in an nonstandard real number set $\mathbf{R}$. We also discuss an application of these dynamical real numbers to cell division and present an interesting intelligent version of the Newton' first law of motion. Finally, in [7], we show that the 'approximate' solution of [6] is in fact generic, in the sense that the more accurate, in fact the exact solution, derived by generating successive self- similar corrections to an initially approximate solution, fails to yield the exact solution even in the limit of infinite number of iterations, thereby proving in a rigorous manner, our original contention that any measurement, even in the classical sense, is intrinsically uncertain.

The new results presented here are the following. Besides restating the above novels features (viz., randomness and time irreversibility) in the context of the new 'exact' but nevertheless intrinsically uncertain solution of [7] in a much more greater detail, we examine the interesting problem of the origin of power law sensitivity in the logistic map at the edge of chaos [10], the $1 / f$ noise of electrical circuites and semiconductor devices [12], and the long tailed hyperbolic type distributions in this extended framework of dynamics.

The paper is organized as follows. We first review the derivation of exact, higher derivative discontinuous $\left(C^{2^{n}-1}\right)$ solutions of eq(1) in Sec.2. The new solution breaks the reflection symmetry $(t \rightarrow-t)$ of the underlying ODE spontaneously [6]. We also show here that besides these finitely differentiable $\left(C^{2^{n}-1}\right)$ time asymmetric solutions as well as the infinitely differentiable, time (reflection) symmetric standard solution, eq(1) possesses another new class of fluctuating solutions which are both infinitely differentiable and time symmetric. Because of these nontrivial classes of finitely and infinitely differentiable fluctuating solutions, a real variable $t$ can undergo changes not only by linear translations, but by inversions $(t \rightarrow 1 / t)$, in the neighbourhood of each real $t$. We next discuss how this defines a nonstandard extension of the real number system [9] in Sec.3. This also clarifies the origin of an intrinsic randomness at as fundamental a level as the real number system. The solutions are intrinsically stochastic with a global ( irreversible) arrow of time (Sec.4). Consequently, every real number is identified with an equivalence class of a continuum of new, infinitesimally separated elements, which are in a state of random fluctuations. In Sec.5, we reinterpret,in the present framework, some new results on unimodal logistic map [10, 11] at the chaos threshold. In particular, we give a derivation of the $q$ exponential power law dynamics of the sensitivity to initial conditions. 
As another application of the late time variability of any dynamical system, arising from the "primordial" (infinitesimal) numerical fluctuations, with generic $1 / f$ spectrum, we discuss $1 / f$ noise in electrical circuits [12] in Sec.6. In Sec.7, we show how a hyperbolic type distribution arises naturally at the asymptotic late time $(t \rightarrow \infty)$ limit even from a normally distributed variate.

\section{Mathematical results}

Because of the novelty of the results, it is instructive to give a fairly complete derivation of such solutions [7]. To this end, let us first construct the solution in the neighbourhood of $t=1$. We need to introduce following notations.

Let $t_{n \pm}=1 \pm \eta_{n}, t_{0} \equiv t, 0<\eta_{n}<<1, \alpha_{n}=1+\epsilon_{n}, n=1,2, \ldots$, and $0<\epsilon_{n}<1$, such that $\epsilon_{n} \rightarrow 0$, as $n \rightarrow \infty$. Next, we write $t_{n \pm}^{\prime}=1 \pm \alpha_{n} \eta_{n}^{\prime}$, so that $\alpha_{n} t_{n-}=t_{n-}^{\prime}$. Consequently, $\eta_{n}^{\prime}=\eta_{n}-\frac{\epsilon_{n}}{\alpha_{n}}$. Here, $\alpha_{n}$ (and $\epsilon_{n}$ ) are arbitrary scaling parameters. As will become clear, natural parametrization is, however, $\epsilon_{n}=\epsilon_{1}^{n}[7]$. Further, $\eta_{n+1}=\alpha_{n}^{2} \eta_{n}^{\prime 2}$.

To construct a nontrivial solution (with the initial condition $\tau(1)=1$ ), we begin with an initial approximate solution in the small scale variable $\eta_{0}$, and then obtain recursively selfsimilar corrections over smaller and smaller scale variables $\eta_{0}^{2}, \eta_{0}^{4}, \ldots$. To this end, let

$$
\tau(t)=\left\{\begin{array}{ll}
\tau_{-} & \text {if } t \lesssim 1 \\
\tau_{+} & \text {if } t \gtrsim 1
\end{array}, \tau_{-}\left(t_{-}\right)=\left(1 / t_{+}\right) f_{1-}\left(\eta_{0}\right), \tau_{+}\left(t_{+}\right)=t_{+}\right.
$$

be an exact solution of eq(1). This is obviously true for the right hand component $\tau_{+}$. Now the same will be true for the nontrivial component $\tau_{-}$provided the correction factor $f_{1-}$ solves exactly the self-similar equation

$$
t_{1-} \frac{\mathrm{d} f_{1-}}{\mathrm{d} t_{1-}}=f_{1-}
$$

in the smaller logarithmic variable $\ln t_{1-}^{-1}$, where $t_{1-}=1-\alpha_{0}^{2} \eta_{0}^{\prime 2} \equiv 1-\eta_{1}$. The exact (nontrivial part of the ) solution could thus be written recursively as

$$
\tau_{-}=C \frac{1}{t_{+}} \frac{1}{t_{1+}^{\prime}} \ldots \frac{1}{t_{(n-1)+}^{\prime}} f_{n-}\left(\eta_{n}^{\prime}\right)
$$

where $f_{n}$ satisfies the $n$th generation self-similar equation

$$
t_{n-} \frac{\mathrm{d} f_{n-}}{\mathrm{d} t_{n-}}=f_{n-}
$$

and $t_{n-}=1-\alpha_{n-1}^{2} \eta_{n-1}^{\prime 2} \equiv 1-\eta_{n}$ so that $\eta_{n}=\alpha_{n-1}^{2} \eta_{n-1}^{\prime 2}$. We also note that $t_{+}^{\prime}=t_{+}$, since $\alpha_{0}=1$. 
Plugging in the initial condition $\tau_{ \pm}=1$ at $t_{ \pm}=1$ (viz., $\eta_{0}=0$ ), one obtains finally the desired solution as

$$
\tau_{-}=C \frac{1}{t_{+}} \frac{1}{t_{1+}^{\prime}} \frac{1}{t_{2+}^{\prime}} \ldots, \tau_{+}=t_{+}
$$

where $C=t_{1+}^{\prime}(0) t_{2+}^{\prime}(0) \ldots$ Notice that $C \neq 1$, since $\eta_{1}^{\prime}=-\epsilon_{1} / \alpha_{1}, \eta_{2}^{\prime}=\epsilon_{1}^{2}-\epsilon_{2} / \alpha_{2}$, etc, when $\eta_{0}=0$. Notice also that it is easy to show that $\left\{f_{n-}\right\}$ is convergent and that $f_{\infty}=\left.\frac{\mathrm{d} f_{n-}}{\mathrm{d} t_{n-}}\right|_{\infty}=$ $\left.\frac{\mathrm{d} \tau}{\mathrm{d} t}\right|_{t=1}=\tau(1)=1$, in the asymptotic limit $n \rightarrow \infty[7]$.

The salient features of this solution are the following.

1. The solution has discontinuous second derivative at $t=1$. The said discontinuity is an effect of an infinity of nonzero rescaling parameters $\epsilon_{n}$. For a finite set of $\epsilon_{n}$ ( or in the special case when $\left.\epsilon_{n}=0, \forall n\right)$, one gets back the standard solution. Moreover, the scale invariance is realized only in a one sided manner. The scaling $\alpha_{n} t_{-}=t_{-}^{\prime}$ does not mean $\alpha_{n} t_{+}=t_{+}^{\prime}$.

2. It also follows that the solution (6) is indeed an exact solution of eq(11) when the ordinary real variable $t=1-\eta_{0}$ (near $\left.t=1\right)$ is replaced by the fat real variable $\mathbf{t}_{-}^{-\mathbf{1}}=\Pi_{0}^{\infty} t_{n+}^{\prime}$. The fat variable $\mathbf{t}$ leaves in $\mathbf{R}$, a nonstandard extension [9] of the ordinary real number set $R$, inhabiting infinitesimal scales (variables) $\ln t_{n}^{\prime} \approx \alpha_{n} \eta_{n}^{\prime}$. All these variables can be treated as independent because of the arbitrary scaling parameters $\epsilon_{n}$.

3. The scaling invariance of eq(1) tells also that, $t=1$ could be realized as $t \rightarrow t / t_{0}=1$, so that the nontrivial solution (6) actually holds in the neighbourhood of every real number $t_{0}$, the 2nd derivative being discontinuous at $t=t_{0}$. Combining the standard and the new solutions together, one can thus write down a more general class of solutions in the form

$$
\tau_{g}(t)=t(1+\phi(t)), \phi(t)=\epsilon t^{-1} \tau\left(t_{1}\right), t_{1}=t / \epsilon
$$

where $\epsilon$ is another arbitrarily small scaling parameter. Note that

$$
t \frac{\mathrm{d} \phi}{\mathrm{d} t}=0
$$

because $\tau$ is an exact solution of eq(1) (one may fix $\epsilon_{n}=\epsilon^{2^{n}}$, in view of remark 2). The 2 nd derivative discontinuity of $\tau$, however, tells that $\phi$ can not be considered simply as an ordinary constant [4, 5] (for an application see Sec.5).

4. Finally, it is easy to verify that eq(11) possesses yet another (nontrivial) class of infinitely differentiable solutions of the form

$$
\tau^{\prime}(t)=\left\{\begin{array}{ll}
\tau_{-}^{\prime} & \text { if } t \geqq 1 \\
\tau_{+}^{\prime} & \text { if } t \gtrsim 1
\end{array}, \tau_{-}^{\prime}\left(t_{-}\right)=\left(1 / t_{+}\right) f\left(\eta_{0}\right), \tau_{+}\left(t_{+}\right)^{\prime}=\left(1 / t_{-}\right) f\left(\eta_{0}\right), f\left(\eta_{0}\right)=\frac{1}{t_{1+}^{\prime}} \frac{1}{t_{2+}^{\prime}} \ldots\right.
$$


which is, however, distinct from the standard solution. This extends the purely fluctuating time symmetric solution studied in ref[6]. Note that the infinite differentiability is restored because of identical self similar corrections in $\tau_{ \pm}^{\prime}$. However, as it should be evident from the above derivations, the iteration schemes for both $\tau_{-}^{\prime}$ and $\tau_{+}^{\prime}$ could be run independently with different sets of scaling factors $\epsilon_{n}$ and $\epsilon_{n}^{\prime}$ respectively, leading again to second derivative discontinuity. Besides these second derivative discontinuous solutions, eq(1), as shown in [7], also accommodates a larger class of $C^{2^{n}-1}$ solutions. Consequently, the simple ode (11) accommodates indeed an astonishingly rich set of solutions belonging to different differentiability classes.

\section{Randomness}

Is randomness a fundamental principle (law) controlling our life and all natural processes? Or, is it simply a projection of our limitations in comprehending such complex phenomena? Is there any well defined boundary separating simple and complex? These and similar related questions on the actual status of randomness are being vigorously investigated in literature [3, 18]. The new class of discontinuous solutions sheds altogether a new light on the ontological status of randomness. (It is believed that randomness in quantum mechanics arises at a fundamental level. However, the Schrõdinger equation, the governing equation of any quantal state, is purely deterministic and time symmetric. The random behaviour is ascribed only through an extraneous hypothesis of a 'collapsed state' at the level of measurement (see, [3] ])

Let us note that eq(1) is the simplest ODE, $t$ being an ordinary real variable. In the framework of the conventional analysis, one can not, in any way, expect, at such an elementary level, a random behaviour in its, so called unique (Picard's), solution. (The chaos and unpredictability could arise only in the presence of explicit nonlinearity in higher order ODEs.) However, the nontrivial scaling, exploited in Sec.2, along with the initial ansatz (2), reveals not only the self similarity of $C^{2^{n}-1}$ solutions over scales $\eta^{2^{n}}$, but also exposes a subtle role of decision making and randomness in generating nontrivial late $t$ behaviour of the solution.

A basic assumption in the framework of the standard calculus is that a real variable $t$ changes by linear translation only. Further, $t$ assumes (attains) every real number exactly. However, in every computational problem within a well specified error bar, a real number is determined only up to a finite degree of accuracy $\epsilon_{0}$,say. Suppose, for example, in a computation, a real variable $t$ is determined upto an accuracy of \pm 0.01 , so that $t$ here effectively stands for the set $t_{\epsilon} \equiv\left\{t \pm \epsilon, \epsilon<\epsilon_{0}=0.01\right\}$ with cardinality $c$ of the continuum. We call $\epsilon$ an 'infinitesimally small' real number (variable). Now, any laboratory computational problem (experiment) is run only over a finite time span, and the influence of such infinitesimally small $\epsilon$ 's, being insignificantly small, could in fact be disregarded. Consequently, the variable $t$ could be written 
near 1 , for instance, as $t_{1}=1+\eta$, where $\eta$ is an ordinary real variable close to 0 , having 'exact' values as long as one disregards infinitesimal numbers $\left(<\epsilon_{0}\right)$ due to practical limitations. At the level of mathematical analysis, such practical limitations being indicative only of natural (physical/biological) imperfections should not jeopardy the existence of an abstract theory of sets, real number system, calculus and so on, shaping the logical framework for an exact and deterministic understanding of natural processes. That such an attempt would remain as an unfulfilled dream is not surprising in view of the new class of discontinuous solutions. As shown in detail in ref[7] ( see also [4, 5, 6]) the $C^{2^{n}-1}$ solutions shows that the real number set $R$ should actually be identified with an nonstandard real number set $\mathbf{R}[9]$ so that every real number $t$ is a fat real (hyperreal ) number $\mathbf{t}$, which means that $t \equiv \mathbf{t}$. Accordingly, a real number $t$ could not be represented simply by a structurless point, but in fact is embedded in a sea of irreducible fluctuations of infinitesimally small numbers $\mathbf{t}=t t_{f}, t_{f}=1+\phi$ denoting universal random corrections from infinitesimals $\phi$ (c.f., eqn(7)). The origin of randomness is obviously tied to the freedom of injecting an infinite sequence of arbitrary scaling parameters $\epsilon_{n}$ into the $C^{2^{n}-1}$ solutions because of scale invariance of eq(1), introducing small scale uncertainty (indeterminacy) in the original variable. (Note that scaling at each stage introduces a degree of uncertainty in the original variable viz., $\delta \eta_{1}=\left|\eta_{1}^{\prime}-\eta_{1}\right|=\epsilon_{1} / \alpha_{1}$ and so on.) It is also shown that the set of infinitesimals $\mathbf{0}=\{ \pm \phi\}$ has a Cantor set like structure viz., discrete, dust like points separated by voids of all possible sizes- and having the cardinality $2^{c}[4,[5]$. Accordingly, an infinitesimal variable could change (within an infinitesimal neighbourhood of a point, say, 1) only by discrete jumps (inversions ) of the form $t_{-}=t_{+}^{-\alpha}, t_{ \pm}=1 \pm \phi$, to cross the gulf of emptiness, length of jumps being arbitrary because of an arbitrary $\alpha$. Note that, the value of a small real number $\eta_{0}$ is uncertain not only upto $\mathrm{O}\left(\eta_{0}^{2}\right)$, but also because of the arbitrary parameter $\epsilon$. Note also that the solution (6) proves explicitly that inversion is also a valid mode of change for a real variable, at least in an infinitesimal neighbourhood of an ordinary real number. We note further that two solutions $\tau_{g}$ and $\tau_{s}$ are indistinguishable for $t \sim \mathrm{O}(1)$ and $\eta_{0}^{2}<<1$. However, for a sufficiently large $t \sim \mathrm{O}\left(\epsilon^{-1}\right)\left(\equiv \mathrm{O}\left(\eta_{0}^{-2}\right)\right)$, the behaviours of two solutions would clearly be different. Finally, the order of discontinuity could be "controlled" by an application of an intelligent decision invoking a nonzero $\epsilon_{n} \neq 0, \epsilon_{m}=0, m<n$ only at the $n$th level of iteration. This freedom of decision making could either be utilized at a pretty early stage of iterations, for instance, $n=1$, say, making the system corresponding to the ode (1) fully intelligent, or be postponed indefinitely $(n=\infty)$ reproducing the standard Picard's solution for a material ( non-intelligent) system [6]. We note that this randomness and potential intelligence being intrinsic properties of the solutions of eq(11) are indelibly rooted to the real number system and hence could not simply be interpreted as due to some coarse graining effect 
analogous to thermodynamics and statistical mechanics. Randomness at a fundamental level arises also in the E- infinity theory [13] ( for other approaches, see [3]).

Before closing this section, let us restate the point of view that is being emerged out of our analysis. Because of historical reasons, current thought processes in any scientific fields of research - physical, chemical, biological, financial, sociological, ... is largely shaped by the role model- Physics(!), which conceives nature as composed purely of inanimate matter and force fields. Consequently, life together with its complex ramifications get reduced to the level of derived concepts - coarse-grained, in some sense, from 'more fundamental'(?) material principles (recall the present state of art of the main-stream biological (biophysical) research!). Even in a purely (lifeless) physical world the origin of randomness from some sort of coarse-graining presupposes our practical limitations in exact measurability (in classical sense) (as in thermodynamics and statistical mechanics, see above). Hence both life-like properties and randomness seem to have been relegated to the level of secondary derived concepts, arisen out purely of inanimate material principles and /or "our ignorance" [18] in comprehending the world, rather than being linked directly to some intrinsic dynamical properties (aspects and/or quality) of life (and nature) itself [3]. This state of affairs does not yet have undergone any dramatic changes with the introduction, as remarked already, of quantum mechanics and quantum field theories, because, the status of randomness in a quantal theory is not yet clear. The new mathematical results explored here ( and in [4]-[7]) would likely to have some profound implications in this regard. With intelligence (and decision making) emerging as a fundamentally new ingredient (degree of freedom) from the mathematical analysis, the traditional framework of a physical theory viz., space, time, matter, energy ( or in a relativistic theory, spacetime and energy) might, in future, be extended and replaced by a truly dynamical framework consisting of intelligence, space, time, matter, energy as envisioned in Ref.[3]. To explore the dynamic properties of the new solutions further, we now examine the origin of time's arrow in the following.

\section{Reflection symmetry breaking and time}

It is well known that time is directed, that is to say, we all have a sense of a forward moving time. The problem of time asymmetry [1] points to a fundamental dichotomy between the (Newtonian) 'time' in physics and mathematics and that of our (objective) experiences. The Newtonian time is non-directed. There is no way to distinguish between a space like variable $x$ with a time variable $t$. Further, all the fundamental equations of physics are time reversal symmetric. However, the existence of $C^{2^{n}-1}$ solutions of eq(1) presents us with a new scenario! One is now obliged to re-examine the conventionally accepted standard notions under this new light. As mentioned already, we show here that time does indeed has an arrow, which is 
inherited, not only by all (physical / biological / social) dynamical systems, but it is also indelible inscribed even to a real number. The concept of time thus turns out to be more fundamental compared to space and may even be considered at par with the real number system (and hence to the existence of intelligence as a fundamental entity)!

To see how a time sense is attached to $C^{2^{n}}$ solutions, we recall first that the variable $t$ is, in general, non-dynamical, and need not denote the (forward) flow of time. In fact, it simply behaves as a labeling parameter. Further, the inversion $t_{-} \leftrightarrow t_{-}^{-1}=t_{+}$may at most be considered as a reversible random fluctuation between $t_{ \pm}$(for a given $\eta>0$ ) with equal probability $1 / 2$. In the usual treatment of ordinary calculus and classical dynamics, $t$ is a non-random ordinary variable, and the above inversion reduces to the symmetry of eq(1) under reversal of sign (parity) $t \rightarrow-t$. The infinitely differentiable standard solution $\tau_{s}(t)$, written (in the notation of eq(2)) as $\tau_{s-}=t_{-}, \tau_{s+}=t_{+},\left(\tau_{s}(1)=1\right)$ is obviously symmetric under this reversible inversion $(\eta \rightarrow-\eta)$. The nontrivial solution $\tau(t)$ in eq(高), however, constitutes an explicit example where this parity invariance is dynamically broken, viz.; when the inversion is realized in an irreversible (one-sided, directed) sense.

To state the above more precisely, let $P: P t_{ \pm}=t_{\mp}$ denote the reflection transformation near $t=1(P \eta=-\eta$ near $\eta=0)$. Clearly, eq(1) is parity symmetric. So is the standard solution $\tau_{s \pm}=t_{ \pm}\left(\right.$since $\left.P \tau_{s}=\tau_{s}\right)$. However, the solution (6) breaks this discrete symmetry spontaneously: $\tau_{-}^{P}=P \tau_{+}=t_{-}, \tau_{+}^{P}=P \tau_{-}=C \frac{1}{t_{-}} \frac{1}{t_{1+}^{\prime}} \frac{1}{t_{2+}^{\prime}} \ldots$, which is of course a solution of eq(11), but clearly differs from the original solution, $\tau_{ \pm}^{P} \neq \tau_{ \pm}$.

To see more clearly how this one-sided inversion is realized, let $t \rightarrow 1^{-}$from the initial point $t \approx 0$. Then at $a$ point in the infinitesimal neighbourhood of $t_{-} \lesssim 1$, the solution $\tau_{-}$carries (transfers) $t_{-}$instantaneously to $t_{+}$by an inversion $\tau_{-} \approx 1 / t_{+}$(we disregard here the $\mathrm{O}\left(\eta_{0}^{2}\right)$ and lower order self similar fluctuations) and subsequently the solution follows the (standard) path $\tau_{+}=t_{+}$in the small scale variable $\eta \gtrsim 0$, as it is now free to follow the standard path till it grows to $\mathrm{O}(\lesssim 1)$, when second order transition to the next smaller scale variable by inversion becomes permissible: $\eta=1 / \eta_{+}, \eta_{+}=1+\bar{\eta}, \bar{\eta} \gtrsim 0$, and so on. Clearly, the generic pattern of (irreversible) (time asymmetric) evolution in $\tau(t)$ over smaller and smaller scales resembles more and more closely the infinite continued fraction of the golden mean: $\tau_{-}(t)=t, 0<t \uparrow \lesssim 1, \rightarrow \tau_{-}=1 /(1+\eta), 0<\eta \uparrow \lesssim 1, \rightarrow \tau_{-}=1 /(1+1 /(1+\bar{\eta})), \bar{\eta} \gtrsim 0$, and so, $\tau_{-}(t) \rightarrow \nu, \nu=(\sqrt{5}-1) / 2$, the golden mean, as $t \rightarrow \infty$. Here, $t \uparrow$ means that $t$ is an increasing variable. We note that the (macroscopic) variable $t$ is reversible as long as $t \sim 1$. Subsequently, this parity symmetry is broken by a random inversion, leading the evolution irreversibly to a smaller scale $0<\eta<<1$, so that $\eta$ has only one way to change (viz., $\eta \rightarrow 1^{-}$), since the transition $t_{+} \rightarrow t_{-}$( the parity reversed solution) is improbable (Note that the growth 
of $\eta_{0}$ in this case is obstructed due to divergence of $1 / t_{-}$as $\eta_{0} \rightarrow 1^{-}$). We also note that the solution which is written only in the neighbourhood of $t=1$, could be extended to the above more general pattern of a 'time irreversible' solution, accommodating an infinite (discrete) set of points where the higher order smoothness of this general function meets obstructions in the form of 2 nd (or higher) derivative discontinuity, forcing it to change stochastically its path down the cascades of the infinite continued fraction of the golden mean. Consequently, time is indeed rediscovered as one with its true elements viz., stochastic irreversibility, rather than being considered simply as a labeling parameter. Analogous ideas are also advocated by Prigogine [18] and El Naschie [13, 14].

The purely fluctuating solution eq(9) is, however, reflection (time) symmetric, as could be verified easily. However, because of intrinsic randomness, this solution would also fail to return an initial value exactly, viz., $t_{-} \rightarrow t_{+} \rightarrow t_{+}^{\prime}, t_{+}^{\prime}$ being only approximately equal to $t_{-}$. This slight mismatch between the initial and final values would definitely induce a symmetry breaking, thereby realizing the time asymmetric solution in due course. Consequently, a variable $\tau$ is intrinsically time-like if and only if it corresponds to the solution (6) which breaks the reflection symmetry spontaneously. Obviously, this definition also applies to the fluctuating solution (9) with different sets of scaling parameters breaking again the reflection symmetry of the underlying equation.

\section{Logistic Maps}

Let us now point out an interesting application of the above formalism. There have been some new, previously unexposed, asymptotic scaling properties of the iterates of unimodal logistic maps at the edge of chaos [10, 11]. For definiteness, we consider here only the dynamics of the sensitivity $\xi_{t}$ to the initial conditions for large iteration time $t$, at the chaos threshold $\mu=\mu_{\infty}=1.40115 \ldots$ of the map $f_{\mu}(x)=1-\mu|x|^{2},-1 \leq x \leq 1$ (for notations see [10]). For a sufficiently large $t$, the ordinary exponential behaviour of sensitivity gives away to a power law behaviour, and is shown to have the $q$-exponential form given by

$$
\left.\xi_{t}=\exp _{q}\left(\lambda_{q} t\right) \equiv\left[1+(1-q) \lambda_{q} t\right)\right]^{1 /(1-q)}
$$

The standard exponential dependence $\xi_{t}=\exp \left(\lambda_{1} t\right)$ is retrieved at the limit $q \rightarrow 1$. The system is said to be strongly insensitive (sensitive) to initial conditions if $\lambda_{1}<0\left(\lambda_{1}>0\right)$. The behaviour, however, gets altered at the edge of chaos. Using the Feigenbaum's RG doubling transformation $\hat{R} f(x)=\alpha f(f(x / \alpha)) n$ times to the fixed point map $g(x)$ viz., $g(x)=\hat{R}^{n} g(x) \equiv \alpha^{n} g^{2^{n}}\left(x / \alpha^{n}\right)$, the values of the $q$-Lyapunov coefficient $\lambda_{q}$ and $q$ are determined to be $\lambda_{q}=\ln \alpha / \ln 2(>0), q=1-\ln 2 / \ln \alpha(<1), \alpha$ being one of the universal 
Feigenbaum constants, $\alpha=2.50290 \ldots$.. Consequently, the critical dynamics corresponds to weak chaos. Notice that the dynamics at the chaos threshold, being the most prominent and readily accessible to numerical experiments, among the critical points of a quadratic map, reveals a universal concerted behaviour, described by the fixed point solution of the RG doubling transformation. One, however, needs to explain the origin of weak chaos, i.e., the system being weakly sensitive, rather than being only weakly insensitive, to initial conditions, at the chaos threshold, when the system approaches the threshold from the left through period doubling route ( for $\mu<\mu_{\infty}$ ).

To reinterpret above observations in the present context, we note first of all that the fixed point equation $\tilde{g}(x)=g(x)$ where $\tilde{g}(x)=\alpha g(g(x / \alpha))$, is a solution of eq(1), viz., $d \tilde{g} / d g=\tilde{g} / g$. Consequently, one expects that the critical dynamics of unimodal quadratic maps at the onset of chaos would be linked directly to our nontrivial solutions to eq(11). As an explicit example, let us investigate the origin of the q-exponential type power law dynamics from the ordinary exponential one

$$
\frac{\mathrm{d} \xi}{\mathrm{d} t}=\lambda_{1} \xi
$$

As the critical point is approached from left (say), the Lyapunov exponent $\lambda_{1} \rightarrow 0^{-}$, and hence gets replaced, in our extended framework, by a 'dynamic' infinitesimal (ordinary zero is an equivalence class of infinitesimals) of the form $\lambda_{1} \rightarrow-\epsilon \lambda_{p} \phi\left(t_{1}\right)$, where $\epsilon(>0)$ is an infinitesimal $\left(\epsilon \neq 0, \epsilon^{2}=\mathrm{O}(0)\right)$ scaling parameter, $\phi=t_{1} \tau\left(t_{1}^{-1}\right)\left(t_{1}=\epsilon t\right)$, and $\lambda_{p}>0, p=p(\epsilon)$ are two generalized constants (c.f., eq( $(8))$ ), both of $\mathrm{O}(1)$. However, when the variation in the intrinsic time like variable $\phi$ becomes relevant at the scale $t \sim 1 / \epsilon$, that of $\lambda_{p}$ would be relevant only at a longer scale viz., $t \sim 1 / \epsilon^{2}$ or more and could be considered as an ordinary constant. The reason for introducing the (third) generalized constant (again with a slower variation than $\phi$ ) $p$ will become clear below. At a critical point, $\mathrm{Eq(11)}$ now reduces to the $\mathrm{RG}$-like equation

$$
t_{1+} \frac{\mathrm{d} \xi}{\mathrm{d} t_{1+}}=\lambda_{p} \xi
$$

To explain the derivation of the above equation let us proceed in steps.

(i) The rescaled variable $t_{1}$, defined by $t_{1}=\epsilon t$, is $\mathrm{O}(1)$ when $t \sim \mathrm{O}(1 / \epsilon)$. Consequently, the critical dynamics would be revealed only at a sufficiently long time scale $t_{1-}=1-\eta \rightarrow 1^{-}$i.e., $t \rightarrow(1 / \epsilon)^{-}$. That means the dynamics at the chaos threshold needs to be probed in an extended framework, viz., in the sense of a limit as the control parameter $\mu$ approaches $\mu_{\infty}$ from left (say) through period-doubling cascade instead of simply replacing $\mu$ by $\mu_{\infty}$ in the map and then iterating. In any computational problem, this extended framework is automatically realized, because of the inherent finite bit (decimal) representation of a real number, such as $\mu_{\infty}$, exact 
value of which could only be approached recursively by increasing its accuracy. The infinitesimal $\epsilon$ (along with an $\mathrm{O}(1)$ variability as represented by $\lambda_{p} \phi\left(t_{1}\right)$ ) then simply corresponds to the infinite trailing bits in the finitely represented real number e.g., $\mu_{\infty}$.

(ii) The critical point equation (12) follows when one makes use of the relation $d \ln t_{1-}=$ $-d \ln t_{1+}$, which is valid for infinitesimal $\eta$ with $\mathrm{O}\left(\eta^{2}\right)=0$ [6]. Clearly, the solution to the above equation is $\xi=\left[1+p \lambda_{p} \eta\right]^{1 / p}$ which corresponds exactly to the $q$ exponential (10) provided we choose $q=1-p, p \lambda_{p}=1$.

(iii) The $q$ exponential solution is, however, valid not only in the 'infinitesimal' neighbourhood of $t_{1}=1$, but for arbitrarily large $t_{1}$, because of the scale invariance of eq(12). Indeed, writing $2^{n} t_{1}=1+\tilde{t}$, the $q$ exponential sensitivity takes the form $\xi=\left[1+(1-q) \lambda_{q} \tilde{t}\right]^{1 /(1-q)}, \tilde{t}$ being large.

(iv) The scale factors $2^{n}$ correspond to the times to determine the trajectory positions $x_{2^{n}}$ of the logistic map with an initial position $x_{i n-}[10]$. It follows therefore that the ratio of the sensitivities at times $2^{n}$ and $2^{n+1}$ viz., $\frac{\xi_{2^{n+1}}}{\xi_{2^{n}}}=\left(\frac{2^{n} t_{1}}{2^{n+1} t_{1}}\right)^{1 / p}=\alpha$ when $p$ remains constant at $1 / p=\ln \alpha / \ln 2$ upto a time $T \approx 2^{N}, N \approx\left|\ln \xi_{\text {in }} / \ln \alpha\right|, \xi_{\text {in }}$ being the initial value of sensitivity, corresponding to the initial iterate $n=0$.

(v) Indeed, to obtain the later estimate, we note that $\xi_{\text {in }}^{p}=t_{1}$, which follows from eq(12). For a non-zero (sufficiently large) $n$, it now follows that $\xi_{n} \equiv\left(2^{n} \xi_{i n}^{p}\right)^{1 / p}=\alpha^{n}$ which translates to $p=\frac{\ln 2}{\ln \alpha}\left(1+\frac{\ln \xi_{i n}}{n \ln \alpha}\right)$. Consequently, a possible variation in $p$ would be revealed only when $n \approx\left|\ln \xi_{\text {in }} / \ln \alpha\right|$, as claimed. Note that $q$ exponential form in the neighbourhood of $t_{1}=1$ belongs to the class of solutions (9) provided the generalized constant $p$ is given by $1 / p=$ $\ln f / \ln t_{1}-1,\left(d p / \ln t_{1}\right)=0$.

The change in sensitivity from strongly insensitive case $\left(\lambda_{1}<0\right)$ to weakly sensitive $\left(\lambda_{q}>\right.$ $0, q<1$ ) power law behaviour is thus explained as an effect of nontrivial infinitesimals and associated inversion $t_{1-} t_{1+} \approx 1$ in the infinitesimal neighbourhood of $t_{1}=1$. The origin of Feigenbaum's constant $\alpha$ ( notice our use of $\xi_{n}=\alpha^{n}[10,11]$ in the above derivation) in the present formalism along with other relevant issues will be considered elsewhere. Another interesting problem is to identify the golden mean number $\nu$ in the critical dynamics.

\section{$61 / f$ spectrum}

The relevance of higher derivative discontinuous solutions to the origin in $1 / f$ noise problem have been discussed in detail in [4, 5]. We note here that in the extended framework of a dynamical theory, accommodating these solutions, any physical, variable $t$, say time, is replaced by $t^{1+\sigma}, \sigma=\ln (1+\phi) / \ln t$ (c.f., Sec.3) where $\sigma$ typically is small $\mathrm{O}(\epsilon)$ for any $\epsilon>0$. The nonzero exponent $\sigma$ introduces a small stochastic fluctuations over the ordinary (time) variable 
t. Clearly, these small scale stochastic fluctuations, existing purely in the real number system, would remain insignificant for any terrestrial (laboratory) inanimate system which persists over a moderate time scale, such as the motion of a (classical) particle under gravity. However, even for simple electrical circuits where the voltage fluctuation spectrum $S_{V}(f)$ is known to vary proportionally with the thermal fluctuation spectrum $S_{T}(f)$, the origin of $1 / f$ noise as observed in [12] could be naturally ascribed to the $C^{2^{n}-1}$ solutions of

$$
c \frac{\mathrm{d} T}{\mathrm{~d} t}=-g\left(T-T_{0}\right)
$$

This equation describes the macroscopic (equilibrium) variations of the temperature $(T)$ of a resistive system with heat capacity $c$, coupled through a thermal conductance $g$ to a heat source at temperature $T_{0}$ [12]. According to the conventional knowledge one does not expect $1 / f$ spectrum from such a simple, purely deterministic, linear uniscale system. One needs, in fact, to consider extraneous nonlinear effects from environment to explain the origin of the generic $1 / f$ fluctuations.

However, according to the present analysis, even this simple system would behave stochastically because of small scale, intrinsic fluctuations in the time variable $t$. These scale free fluctuations could influence the late time behaviour of the system provided the system is 'allowed' to survive over a period $t>>1 / \epsilon, \epsilon=g / c$. Notice that ordinarily a system following purely eq(13) is assumed to relax to the equilibrium temperature $T_{0}$ after $t \approx 1 / \epsilon$. The late time variability that is observed in any resistive system is then ascribed normally to the complex nature of the resistive medium and/or (nonlinear) interactions with environment [12], asking for an explicit modeling. The generic observation of $1 / f$ fluctuations in metal films and semiconductors still eludes a universal explanation for its microscopic origin in the framework of conventional dynamical theories [15].

In view of $C^{2^{n}-1}$ solutions, we now have an extended framework to re-examine the above problem. The solution of eq(13) now have the form $T(t)-T_{0}=t^{\sigma} e^{-\epsilon t}, \sigma$ being a small fluctuating variable. As noted already, this random exponent would lead to small scale stochastic modulations over the (mean) macroscopic decay mode, as observed in physical systems. These small scale (power law) fluctuations would persists even far beyond the ordinary relaxation time. Accordingly, a time series of temperature fluctuations $\left(T_{f}(t)=\left(T(t)-T_{0}\right) e^{\tau}\right)$ recorded over a period of a few decades ( 1 to $10^{4}$, say), in the unit of the dimensionless time $\tau=\epsilon t$, would reveal a scale free $1 / f$ type variability. For, the two point autocorrelation function of this intrinsic fluctuations has the form $C(t)=<T_{f}(t) T_{f}(0)>=c<T_{f}(t)>=c<t^{\sigma}>\approx c t^{<\sigma>}, T_{f}(0)=c$, where $\langle\sigma\rangle$ is the expectation value of the random exponent and $c \sim \mathrm{O}(1)$ is the initial (background) noise in the system. The associated probability distribution would have a generic late 
$t$ behaviour, resembling infinitely divisible Levi type distributions [19] (see below). The corresponding power spectrum of this stochastic scale free fluctuation is given by $S(f) \sim 1 / f^{1-<\sigma>}$. We note that an arbitrarily small nonzero $\sigma$ is sufficient to generate a $1 / f$-like fluctuation. In other words, intrinsically random, infinitesimal scales associated with the time variable $t$ could act as a perennial source of small scale fluctuations leading to the universal low frequency $1 / f$ spectrum. However, an accurate determination of the intensity of the fluctuations (viz, the constant of proportionality in the observed spectrum $S_{V}(f) \propto V^{2} / f^{a}, a \approx 1$ ) may require further work [12, 15]. The relevance of number theory to $1 / f$ noise problem is also pointed out by Planat [16]. El Naschie suggested that the exponent $\beta$ of the $1 / f^{\beta}$ noise to semiconductors would be related to the golden mean in the framework of the E-infinity theory [17].

\section{Hyperbolic distribution}

In ref [4] we show that the scale free infinitesimal fluctuations follow a nongaussian, BramewellHoldsworth-Pinton (BHP) [20] distribution. Here we show how a hyperbolic, power law tail gets superposed generically in any distribution when the concerned random variate is assumed to leave in $\mathbf{R}$. To see this it suffices to consider only a normally distributed variate, because by the central limit theorem a normal probability density acts as the attractor for any probability density with finite moments. Let $t$ be a zero mean normal variate, with unit standard deviation. The corresponding fat variate could be written as $\mathbf{t}^{2}=t^{2}+\phi, \phi=\epsilon(t) \ln t^{2}$, being a random infinitesimal satisfying eq(8) in the logarithmic variable $\ln t$ (c.f., Appendix). Consequently, the normal density function $\propto e^{-t^{2} / 2}$ gets a generic power law tail $t^{-\epsilon} e^{-t^{2} / 2}$. This generic power law tail in the present extended formalism should become important in the future studies on the statistics of rare events. We close with the remark that occasional detections of exceptional events in an experiment of, for instance, a normal variate over a prolonged period could be explained by this slowly varying tail. Note that the power law variability would become visible only in the asymptotic limits $(t \rightarrow \pm \infty)$ because of an infinitesimal $\epsilon$, so that $\phi$ remains vanishingly small in any laboratory experiments over a finite time scale.

\section{Appendix}

A continuously differentiable function $f(t)$ of a real variable could be defined as an integral of the ODE $\frac{\mathrm{d} x}{\mathrm{~d} t}=f^{\prime}(t)$. For the gaussian $e^{-t^{2} / 2}$, the relevant equation is $\frac{\mathrm{d} x}{\mathrm{~d} t}=-t e^{-t^{2} / 2}$, and hence the corresponding hyperreal (fat) extension is given by $\mathbf{t}^{2}=t^{2}+\epsilon(t) \ln t^{2}$. The extension of the linear variable $t$ is given by $\mathbf{t}=t+\epsilon(t) \ln t$. The extended exponential $e^{\mathbf{t}}=t^{\epsilon} e^{t}$ would therefore have a slowly fluctuating power law tail. Note that the variable $t$ gets the infinitesimal 
correction term in the logarithmic variable $\ln t$, when the infinitesimal $\epsilon$ satisfies the equation

$$
\sigma \frac{\mathrm{d} \epsilon}{\mathrm{d} \sigma}=-\epsilon, \sigma=\ln t
$$

\section{References}

[1] H D Zeh, The Direction of Time, Springer, Berlin (1992).

[2] W H Press, Comments Astrophys. 7103 (1978).

[3] P. Grigolini, P. Hamilton, J. Roberts and B. J. West, Towards a new vision of complexity, Chaos, Solitons \& Fractals, 20, (2004), 1-185 (special issue).

[4] D P Datta, A new class of scale free solutions to linear ordinary differential equations and the universality of the Golden Mean $\frac{\sqrt{5}-1}{2}=0.618033 \ldots$.. Chaos, Solitons $\&$ Fractals, 17, (2003), 621-630.

[5] D. P. Datta, The golden mean, scale free extension of real number system, fuzzy sets and 1/f spectrum in physics and biology, Chaos, Solitons $\mathscr{E}$ Fractals, 17, (2003), 781.

[6] D. P. Datta, Dynamical real numbers and living systems, Chaos, Solitons \& Fractals, 20, (2004), 705-712.

[7] D. P. Datta and M. K. Bose, Higher derivative discontinuous solutions to linear ordinary differential equations: a new route to complexity? Chaos, Solitons 83 Fractals, 22, (2004), 271.

[8] H. Weiss and V. Weiss, The golden mean as clock cycle of brain wave, Chaos, Solitons 86 Fractals, 18, 2003, 643-652.

[9] A Robinson, Nonstandard analysis, North-Holland, Amsterdam, (1966). the edge of chaos, Phys. Rev. E, 65, (2002), 036207.

[10] F. Baldovin and A. Robledo, Universal renormalisation group dynamics at the onset of chaos in logistic maps and nonextensive statistical mechanics, Phys. Rev. E, 66,(2002), $045104(\mathrm{R})$.

[11] F. Baldovin and A. Robledo, Exact renormalisation group analytical results for the dynamics at the edge of chaos of the logistic map, LANL preprint archive cond-mat/0304410.

[12] R. F. Voss and J. Clarke, Flicker (1/f) noise: equilibrium temperature and resistance fluctuations, Phys. Rev. D, 13, (1976), 556. 
[13] M. S. El Naschie, A review of E-infinity theory and the mass spectrum of high energy particle physics, Chaos, Solitons \& Fractals, 19 (2004), 209-236.

[14] M. S. El Naschie, Time symmetry breaking, duality and Cantorian spacetime, Chaos, Solitons \& Fractals, 7, (1996), 499.

[15] M Planat, Class numbers in imaginary quadratic field and the $1 / f$ noise of an electron gas, CNRS (Paris) preprint (2002). .

[16] M. Planat, 1/f noise, the measurement of time and number theory, Fluctuation and Noise Lett. 1,(2001), R56.

[17] M. S. El Naschie, Penrose tiling, semi-conduction and Cantorian $1 / f^{\alpha}$ spectra in four and five dimensions, Chaos, Solitons \& Fractals, 1993, 3, 489-491.

[18] I Prigogine, The arrow of time, available at http://www.icra.it/Publications/Books/Prigogine /Prigogine_motivation.htm

[19] B. J. West and W. Deering, Fractal physiology for physicists: Levy statistics, Phys. Repts., 246, (1994), 1.

[20] S T Bramwell, K. Christensen, J -Y Fortin, P C W Holdsworth, H J Jensen, S Lise, J M Lopez, M Nicodemi, J -F Pinton, and M Sellitto, Phys. Rev.Lett. 84 3744, (2000). 